\title{
Force Analysis of the Overconstrained Mechanisms Based on Equivalent Stiffness Considering Limb Axial Deformation
}

Jinwei Guo

Yanshan University

Yongsheng Zhao

Yanshan University

Bo Chen

Yanshan University

Guoxing Zhang

Yanshan University

Yundou Xu ( $\nabla$ ydxu@ysu.edu.cn )

Yanshan University

Jiantao Yao

Yanshan University

Original Article

Keywords: Active overconstrained, Passive overconstrained, Passive-input overconstrained, Equivalent stiffness, Force distribution

Posted Date: July 19th, 2021

DOl: https://doi.org/10.21203/rs.3.rs-634709/v1

License: (c) (i) This work is licensed under a Creative Commons Attribution 4.0 International License.

Read Full License 
Force analysis of the overconstrained mechanisms based on equivalent stiffness considering limb

\author{
axial deformation \\ Jinwei Guo ${ }^{1}$, Yongsheng Zhao ${ }^{1,2}$, Bo Chen ${ }^{1}$, Guoxing Zhang ${ }^{1}$, Yundou $\mathrm{Xu}^{1,2, *}$, Jiantao Yao ${ }^{1,2}$ \\ 1. Parallel Robot and Mechatronic System Laboratory of Hebei Province, Yanshan University, Qinhuangdao 066004, \\ China
}

2. Key Laboratory of Advanced Forging \& Stamping Technology and Science of Ministry of National Education, Yanshan

University, Qinhuangdao 066004, China

\begin{abstract}
Considering the limb axial deformation, taking typical 2SS+P and 7-SS passive overconstrained mechanisms, 2SPS+P and 7-SPS active overconstrained mechanisms, and 2SPS+P and 7-SPS passive-input overconstrained mechanisms as examples, a new force analysis method based on the idea of equivalent stiffness is proposed. The equivalent stiffness matrix of passive overconstrained mechanism is derived by combining the force balance and deformation compatibility equations with consideration of axial elastic limb deformations. The relationship between the constraint wrench magnitudes and the external force, limb stiffness is established. The equivalent stiffness matrix of active overconstrained mechanism is derived by combining the force balance and displacement compatibility equations. Here, the relationship between the magnitudes of the actuated wrenches and the external force, limb stiffness is investigated. Combining with the equivalent stiffness of the passive overconstrained mechanism, an analytical relationship between the actuated forces of passive-input overconstrained mechanism and the output displacement, limb stiffness is explored. Finally, adaptability of the equivalent stiffness to overconstrained mechanisms is discussed, and the effect of the limb stiffness on overconstrained mechanisms force distribution is revealed.
\end{abstract}

Keywords: Active overconstrained; Passive overconstrained; Passive-input overconstrained; Equivalent stiffness; Force distribution

\title{
1. Introduction
}

In recent years, overconstrained mechanism has become one of the research hotspots in processing and manufacturing industries. Its applications involve areas such as deployable antennas, machine tools, robots, medical equipment and entertainment facilities. According to the different generation methods of overconstraints and actuated modes, overconstrained mechanism is divided into passive overconstrained mechanism [1-3], active overconstrained mechanism [4-7] and passive-input overconstrained mechanism [8].

Passive overconstrained mechanism indicates that various constraints are imposed on the moving platform via several limbs. Some of limbs may overlap with another system constraint, or may contain common or redundant

*Corresponding author. Tel./Fax: +86-13613342823. E-mail address: ydxu@ysu.edu.cn (Y.D. Xu). 
mechanism constraints. Passive overconstrained parallel mechanism is characterized by a simple structure, high rigidity, high precision, and an important role in heavy load applications. Common constraints or redundant constraints have no effect on the motion characteristics of the mechanism. However, their utilization results in a static indeterminate problem when conducting force analyses. This, in turn, increases the relative complexity and difficulty when conducting analyses for this type of mechanism. Bi et al. [9] established force balance equations for all components of passive overconstrained parallel mechanism 2UPR+SPR via Newton-Euler method. The authors obtained a complete and solvable dynamic model by combining deformation compatibility equations. Wojtyra et al. [10-13] proposed several analytical and numerical methods for solving the joint constraint reaction forces of passive overconstrained mechanisms. By considering tension and compression deformation as well as stiffness of the limbs, a solution for the force problem of 7-SS passive overconstrained parallel structure six-dimensional force sensor is obtained [14]. Under the assumption of independent elastic limb deformations when loaded with the actuated force/torque and the constraint force/couple, a force analysis method for passive overconstrained parallel mechanisms is proposed [15]. Based on the screw theory, Huang et al. [16] proposed a force analysis method for passive overconstrained parallel mechanisms with either a collinear overconstrained force or coaxial overconstrained couple. Zhang et al. [17] and Rezaei et al. [18] established stiffness models of passive overconstrained mechanism Exechon and 2PRR-PPR via sub-structure synthesis technology and Karl's theorem, respectively. Based on the second Karl's theorem, Yang et al. [19] deduced the stiffness matrix of the limbs constraint wrenches system of the passive overconstrained parallel mechanism. Wu et al. [20] investigated the influence of structural parameters on dynamic characteristics of PRRRP of overconstrained mechanisms. Arian et al. [21] employed Newton-Euler method to study the kinematics and dynamics of a three-degree-of-freedom (3-DOFs) moving overconstrained mechanism. Yang et al. [22] analyzed the elastic static stiffness of 2PUR-PSR overconstrained mechanism. According to [3], passive overconstrained parallel mechanism can be divided into limb stiffness coupling mechanism and limb stiffness decoupling mechanism. Furthermore, complex space elastic limb deformation is considered to propose a force analysis method for passive overconstrained parallel mechanism. It should be mentioned that additional research on the force analysis of passive overconstrained mechanism is conducted in [23-26].

Active overconstrained mechanism is defined as the mechanism that causes overconstraints due to actuation redundancy, i.e., the number of active inputs being greater than the number of DOFs. The introduction of actuation redundancy has played an important role in avoiding singularities, expanding the working space [4], eliminating the gap of the kinematic joint [27], improving the rigidity of the machine and actuated stability [28], 
and especially improving the bearing load capacity [29-30]. Introduction of redundant actuation results in a statically indeterminate force analysis of active overconstrained mechanism. The actuated force/torque has infinite sets of theoretical solutions, and can be actively allocated in practice according to various optimization goals. Pseudo-inverse method is utilized to distribute the actuated force/torque of the active overconstrained mechanism in [31], with minimum input force/torque of the system being the optimization goal. In order to evaluate the actuated force compatibility performance of active overconstrained parallel mechanism, two indicators are proposed: the actuated force compatibility rate and the compatibility factor [32]. It is worth to mention that additional research on analysis of active overconstrained mechanism is carried out in [33-36].

Passive-input overconstrained mechanism is defined between the active overconstrained mechanism and the passive overconstrained mechanism. Although the overconstraint is generated by redundant actuation, the actuation itself is passive, such as spring, and its actuation mode cannot be actively controlled. Therefore, the force analysis of such mechanism is relatively complicated. Xu et al. [37] investigated mechanical and electrical properties with the goal of optimizing the structure of tetrahedral truss deployable mechanism. Xu et al. [6] established a dynamic model of passive-input overconstrained tetrahedral deployable antenna mechanism based on the Lagrange method. Li et al. [38] investigated the stiffness of the actuation torsion spring, hinge damping, influence of gravity and pretension of cable net to analyze the mechanical characteristics during deployment process of hoop-deployable antenna. Jin et al. [39] established a flexible multi-body dynamics model of a truss deployable antenna. Based on their research of kinematics theoretical model and simulation analysis of truss deployable antenna mechanism, Liu et al. [40] obtained the required actuated force to ensure the smooth deployment of the mechanism, while simultaneously designing the actuated spring.

Force analyses of passive, active and passive-input overconstrained mechanism are all statically indeterminate problems. For special passive-input overconstrained mechanisms, motion force analysis is even more complicated. Currently, researchers have carried out force investigations of the aforementioned three types of overconstrained mechanisms. Although they have achieved certain results, the proposed methods have some limitations and do not distinguish the difference and connection of static indeterminate mechanics. Especially for passive-input overconstrained mechanisms, some methods have been proposed in the existing literature. However, there is no clear conclusion whether the existing methods are applicable to more complex multi-DOF mechanisms $[8,37-40]$.

In this paper, static indeterminate mechanics of three types of overconstrained mechanisms is investigated, compared, and analyzed. This presents an important theoretical contribution for the structural design, control, 
and application of overconstrained mechanisms.

Apart from the introductory chapter, the paper itself is organized as follows. In Section 2, six types of overconstrained mechanisms are considered as an example to explore the force distribution of overconstrained mechanisms based on the idea of equivalent stiffness. The corresponding simulation models are established for simulation verification. In Section 3, the adaptability of equivalent stiffness to different overconstrained mechanisms is demonstrated, and the general expression is provided. In Section 4, influence mechanism of limb stiffness on the force distribution of different overconstrained mechanisms is discussed. In the final section, conclusions based on the conducted research are made.

\section{Force distribution mechanism of different overconstrained mechanisms based on the idea of equivalent stiffness}

The key of the force analysis method of overconstrained mechanism based on the idea of equivalent stiffness is to obtain the equivalent stiffness. Under the action of external force, the output motion of the mechanism is solved based on the equivalent stiffness, and then the force distribution of the mechanism is obtained. Three types of overconstrained mechanisms are taken as examples to solve the equivalent stiffness in detail and reveal the role of the idea of equivalent stiffness in the force analysis of overconstrained mechanisms.

\subsection{Passive overconstrained mechanism}

Taking 2SS+P and 7-SS passive overconstrained structures as examples, the equivalent stiffness and constraint wrench of each limb are solved.

\subsubsection{The 2SS+P passive overconstrained structure}

As presented in Fig. 1, the planar parallel structure 2SS+P consists of both moving and the fixed platform, as well as the two SS limbs and a P limb. Two ends of the limbs 1 and 2 are connected to the moving and the fixed platform via spherical joint (S). The P joint axis of limb 3, as well as axes of limbs 1 and 2 are simultaneously parallel and coplanar. A coordinate system $o-x y z$ is established at the center of the moving platform. The $x$-axis is parallel to the $\mathrm{P}$ joint axis, the $z$-axis coincides with the normal direction of the plane where the structure is located, and the $y$-axis is determined based on the right-hand rule. Each of the two SS limbs has a local DOF which enables the rotation around its own axis. Furthermore, each limb provides a constraint force to the moving platform along the $\mathrm{P}$ joint axis, while the moving platform is subject to two linearly related constraint forces with DOF being zero. Therefore, the $2 \mathrm{SS}+\mathrm{P}$ structure represents a plane passive overconstrained parallel structure. 


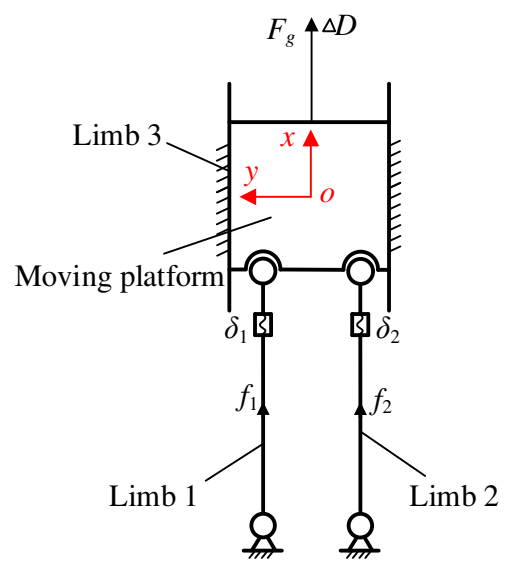

Fig. 1. The 2SS+P passive overconstrained parallel structure

Structure of the $\mathrm{P}$ joint is theoretically rigid, i.e., apart from the $\mathrm{P}$ joint axis direction, the deformation in other directions is zero. Thus, only deformations of two SS limbs under the external force along the direction of P joint is considered. For overconstrained structure $2 \mathrm{SS}+\mathrm{P}$, the main deformation source is limb axial deformation, the axial elastic deformation of limb 1 should be equal to the axial elastic deformation of limb 2 . The axial elastic deformations of limb 1 and limb 2 are designated as $\delta_{1}$ and $\delta_{2}$, respectively, while the micro-displacement of the moving platform is designated as $\Delta D$. In other words, the structure meets the deformation compatibility relationship:

$$
\delta_{1}=\delta_{2}=\Delta D
$$

The constraint forces provided by the two SS limbs are $f_{1}$ and $f_{2}$, respectively. The force balance equation of the structure can be expressed as follows:

$$
\boldsymbol{G} \boldsymbol{f}=F_{\mathrm{g}}
$$

where $\boldsymbol{G}=\left(\begin{array}{ll}1 & 1\end{array}\right), \boldsymbol{f}=\left(\begin{array}{ll}f_{1} & f_{2}\end{array}\right)^{\mathrm{T}}$, and $\boldsymbol{F}_{g}$ represents a generalized external force on the moving platform such as the external load, inertial force, gravity or friction.

The relationship between the axial elastic deformation of the limb and the constraint force can be described as:

$$
\left(\begin{array}{l}
f_{1} \\
f_{2}
\end{array}\right)=\boldsymbol{K}\left(\begin{array}{l}
\delta_{1} \\
\delta_{2}
\end{array}\right)
$$

where $\boldsymbol{K}=\operatorname{diag}\left(k_{1} \quad k_{2}\right)$. Parameters $k_{1}$ and $k_{2}$ represent axial stiffness of two SS limbs, respectively.

By combining Eqs. (1) (3), the following expression can be obtained: 


$$
F_{g}=\boldsymbol{G} \boldsymbol{K} \boldsymbol{G}^{\mathrm{T}} \Delta D=\left(k_{1}+k_{2}\right) \Delta D
$$

According to Eq. (4), the equivalent stiffness of passive overconstrained structure 2SS+P can be expressed as:

$$
K_{\mathrm{e}}=\boldsymbol{G} \boldsymbol{K} \boldsymbol{G}^{\mathrm{T}}=k_{1}+k_{2}
$$

After obtaining the equivalent stiffness of the structure, the micro-displacement of the moving platform can be obtained according to Eq. (4). Deformation of each limb can be obtained according to Eq. (1), and then the magnitude of the constraint wrench for each limb is obtained according to Eq. (3):

$$
\boldsymbol{f}=\boldsymbol{K} \boldsymbol{G}^{\mathrm{T}}\left(K_{e}\right)^{-1} F_{g}
$$

\subsubsection{7-SS passive overconstrained structure}

As depicted in Fig. 2, the six-dimensional force sensor, 7-SS, with parallel structure configuration consists of both moving platform and the fixed platform, as well as seven measuring limbs (denoted as $\left.D_{i} U_{i}, i=1,2, \ldots, 7\right)$. Both ends of each limb are connected with both moving and the fixed platform via $\mathrm{S}$ joint. The reference coordinate systems $O_{\mathrm{d}}-X_{\mathrm{d}} Y_{\mathrm{d}} Z_{\mathrm{d}}$ and $O_{\mathrm{u}}-X_{\mathrm{u}} Y_{\mathrm{u}} Z_{\mathrm{u}}$ are established at the center of the fixed platform and the moving platform, respectively. The angle between the $X_{\mathrm{d}}$-axis and $O_{\mathrm{d}} D_{1}$ is defined as $\alpha_{1}$, while the angle between the $X_{\mathrm{u}}$-axis and $O_{\mathrm{u}} U_{1}$ is defined as $\beta_{1} . Z_{\mathrm{d}}$ and $Z_{\mathrm{u}}$ axes are perpendicular to the plane of the fixed platform. Parameter $R_{\mathrm{u}}$ is the distribution radius of the spherical hinge points of the moving platform, while $R_{\mathrm{d} 1}$ and $R_{\mathrm{d} 2}$ are the distribution radii of the spherical hinge points of the inner ring and the outer ring of the fixed platform, respectively. Parameter $H$ marks the distance between the fixed platform and the moving platform. Angle $\alpha_{2}$ is defined between the line projection of the fourth spherical hinge point of the fixed platform and the origin of the coordinate on the $O X Y$ plane and the $X$-axis of the coordinate system. Angle $\beta_{2}$ represent an angle between the projection of the line between the fourth spherical hinge point of the moving platform and the origin of the coordinate on the $O X Y$ plane and the $X$-axis of the coordinate system.

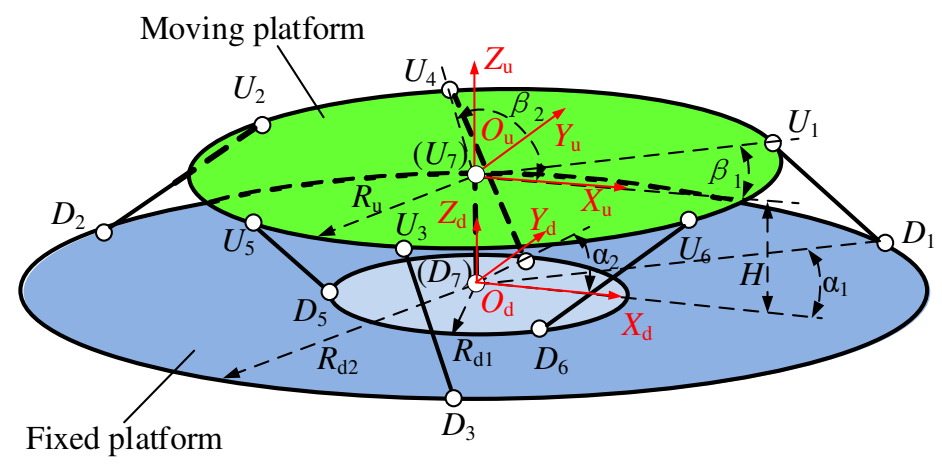

Fig. 2. The 7-SS passive overconstrained structure 
In the 7-SS structure, each limb provides a constraint force along the limb axis to the moving platform, the main deformation source is limb axial deformation. The moving platform is subject to seven linearly related constraint forces. Maximum number of linearly independent variables is six, limiting all the DOFs of the moving platform. According to definition of the overconstrained wrenches, it is observed that these seven constraint wrenches are all characterized as overconstrained wrenches. Thus, the 7-SS structure is defined as spatial passive overconstrained parallel structure. Regardless of the limb gravity, under overconstrained and external forces acting upon the moving platform, the force balance equation of the structure can be expressed as:

$$
\boldsymbol{\$}_{F}=f_{\mathrm{r}, 1} \hat{\boldsymbol{S}}_{\mathrm{r}, 1}+f_{\mathrm{r}, 2} \hat{\boldsymbol{S}}_{\mathrm{r}, 2}+\cdots+f_{\mathrm{r}, 7} \hat{\boldsymbol{S}}_{\mathrm{r}, 7}=\boldsymbol{G}_{f}^{F} \boldsymbol{f}
$$

where $\boldsymbol{S}_{F}=\left(\begin{array}{llllll}F_{x} & F_{y} & F_{z} & M_{x} & M_{y} & M_{z}\end{array}\right)^{\mathrm{T}}$ represents the six-dimensional external force on the moving platform. $\boldsymbol{G}_{f}^{F}=\left(\begin{array}{llll}\hat{\boldsymbol{S}}_{\mathrm{r}, 1} & \hat{\boldsymbol{S}}_{\mathrm{r}, 2} & \mathrm{~L} & \hat{\boldsymbol{S}}_{\mathrm{r}, 7}\end{array}\right), \quad \hat{\boldsymbol{S}}_{\mathrm{r}, i}(i=1,2, \mathrm{~L}, 7) \quad$ represents unit wrenches of the constraint wrenches $\quad \boldsymbol{S}_{\mathrm{r}, i}$. $\boldsymbol{f}=\left(\begin{array}{llll}f_{\mathrm{r}, 1} & f_{\mathrm{r}, 2} & \mathrm{~L} & f_{\mathrm{r}, 7}\end{array}\right)^{\mathrm{T}}, \quad f_{\mathrm{r}, i}$ represents the magnitudes of the constraint wrenches $\quad \boldsymbol{S}_{\mathrm{r}, i}$.

According to the principle of virtual work, the following expression is true:

$$
\boldsymbol{f}^{\mathrm{T}} \Delta \boldsymbol{l}=\boldsymbol{\$}_{F}^{\mathrm{T}} \boldsymbol{D}
$$

where $\Delta \boldsymbol{l}=\left[\begin{array}{llll}\Delta l_{1} & \Delta l_{2} & \cdots & \Delta l_{7}\end{array}\right]^{\mathrm{T}}$ represents deformation of the each limb along the axis, while $\boldsymbol{D}=\left(\begin{array}{llllll}\delta_{x} & \delta_{y} & \delta_{z} & \varphi_{x} & \varphi_{y} & \varphi_{z}\end{array}\right)^{\mathrm{T}}$ represents the six-dimensional micro-displacement of the moving platform under the influence of the external force $\$_{F}$.

By substituting Eq. (7) into Eq. (8), Eq. (9) can be obtained:

$$
\Delta \boldsymbol{l}=\left(\boldsymbol{f}^{\mathrm{T}}\right)^{-} \boldsymbol{\phi}_{F}^{\mathrm{T}} \boldsymbol{D}=\left(\boldsymbol{f}^{\mathrm{T}}\right)^{-} \boldsymbol{f}^{\mathrm{T}}\left(\boldsymbol{G}_{f}^{F}\right)^{\mathrm{T}} \boldsymbol{D}=\left(\boldsymbol{G}_{f}^{F}\right)^{\mathrm{T}} \boldsymbol{D}
$$

Magnitude of the constraint force for each limb can be expressed as:

$$
f=K \Delta l
$$

where $\boldsymbol{K}=\operatorname{diag}\left(k_{1} \quad k_{2} \quad \mathrm{~L} \quad k_{7}\right)$ and $k_{i}$ represents the axial stiffness of the limb.

By substituting Eqs. (9) and (10) into Eq. (1) yields:

$$
\$_{F}=\boldsymbol{G}_{f}^{F} \boldsymbol{K}\left(\boldsymbol{G}_{f}^{F}\right)^{\mathrm{T}} \boldsymbol{D}
$$

According to Eq. (11), the equivalent stiffness of the passive overconstrained 7-SS structure can be deduced as follows:

$$
\boldsymbol{K}_{e}=\boldsymbol{G}_{f}^{F} \boldsymbol{K}\left(\boldsymbol{G}_{f}^{F}\right)^{\mathrm{T}}
$$


After obtaining the equivalent stiffness of the structure, the deformation of the moving platform can be obtained according to Eq. (11). On the other hand, the deformation of each limb can be obtained according to Eq. (9). Lastly, according to Eq. (10), the magnitude of limb constraint force is obtained as:

$$
\boldsymbol{f}=\boldsymbol{K}\left(\boldsymbol{G}_{f}^{F}\right)^{\mathrm{T}}\left(\boldsymbol{K}_{e}\right)^{-1} \boldsymbol{\$}_{F}
$$

\subsubsection{Simulation verification}

7-SS passive overconstrained structure is considered as an example for simulation analysis. As shown in Fig. 3, a rigid-flexible hybrid model with structure parameters provided in Table 1 is established. Each limb link represents a flexible body, while both moving and the fixed platforms represent rigid bodies. Six-dimensional external force $\$_{F}=[10 \mathrm{~N} 25 \mathrm{~N} 20 \mathrm{~N} 15 \mathrm{~N} \cdot \mathrm{m} 20 \mathrm{~N} \cdot \mathrm{m} 5 \mathrm{~N} \cdot \mathrm{m}]^{\mathrm{T}}$ at the center of the moving platform is applied. By assuming negligible gravity effects, theoretical and simulation values of the constraint forces of each limb are compared in Table 2.

Table 1. Structural parameters of 7-SS passive overconstrained structure

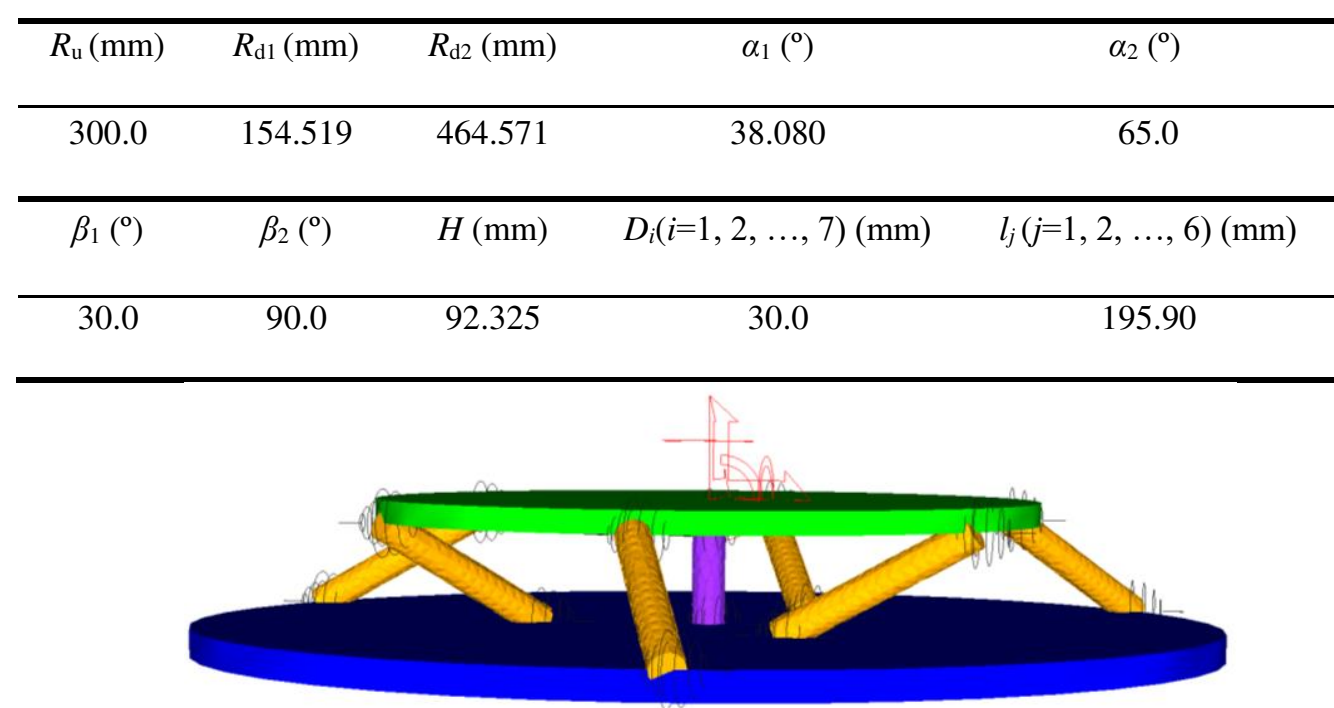

Fig.3 Simulation model of the 7-SS passive overconstrained structure

Table 2. Comparison between theoretical and simulation values of constraint forces

\begin{tabular}{ccccc}
\hline Magnitude of the & $\begin{array}{c}\text { Theoretical } \\
\text { value } / \mathrm{N}\end{array}$ & $\begin{array}{c}\text { Simulation } \\
\text { value } / \mathrm{N}\end{array}$ & $\begin{array}{c}\text { Absolute } \\
\text { error } / \mathrm{N}\end{array}$ & $\begin{array}{c}\text { Relative } \\
\text { error }\end{array}$ \\
\hline$f_{1}$ & -38.5398 & -39.5548 & 1.015 & $2.5661 \%$ \\
$f_{2}$ & 53.7447 & 52.5488 & 1.1959 & $2.2758 \%$ \\
$f_{3}$ & 55.3749 & 55.1765 & 0.1984 & $0.3596 \%$ \\
$f_{4}$ & 24.4308 & 25.4113 & 0.9805 & $3.8585 \%$
\end{tabular}




$\begin{array}{lllll}f_{5} & -33.6477 & -32.4862 & 1.1615 & 3.5754 \% \\ f_{6} & -44.9902 & -44.8267 & 0.1635 & 0.3647 \% \\ f_{7} & 12.2836 & 12.3326 & 0.049 & 0.3973 \%\end{array}$

According to Table 2, the relative error of the constraint forces between the theoretical and the simulation values $f_{1} \sim f_{7}$ does not exceed $3.9 \%$. This, the error is mainly caused by the mesh division method and division accuracy when establishing the flexible body model of each limb. It can be concluded that the force analysis of the passive overconstrained mechanism based on equivalent stiffness method is successfully verified.

\subsection{Active overconstrained mechanisms}

Taking 2SPS+P and 7-SPS active overconstrained mechanisms as examples, the equivalent stiffness and distribution of actuated force of each limb are solved.

\subsubsection{SPS+P active overconstrained mechanism}

The planar parallel mechanism $2 \mathrm{SPS}+\mathrm{P}^{[41]}$ is shown in Fig. 4. The main difference from the planar passive overconstrained mechanism $2 \mathrm{SS}+\mathrm{P}$ is that the $\mathrm{P}$ joints along the limb axes are added to the two existing SS limbs and considered as the actuated joints. In a similar manner to the $2 \mathrm{SS}+\mathrm{P}$ passive overconstrained mechanism, a coordinate system $o$-xyz is established at the center of the moving platform. The $x$-axis is parallel to the $\mathrm{P}$ joint axes of the three limbs, the $z$-axis coincides with the normal direction of the plane where the mechanism is located, and the $y$-axis is determined based on the right-hand rule. The two SPS limbs each have a local DOF that enables their rotation around the P joint axis, which does not provide constraints on the moving platform. Thus, the DOF of the 2SPS+P mechanism is determined by the limbs P. In addition, DOF of the mechanism can also be obtained according to the modified G-K equation:

$$
M=d(n-g-1)+\sum_{i=1}^{g} f_{i}+v-\xi=6(6-7-1)+15-2=1
$$

Eq. (14) indicates that the mechanism 2SPS+P has one DOF and two actuations. Therefore, the 2SPS+P the mechanism is considered as a planar active overconstrained parallel mechanism. 


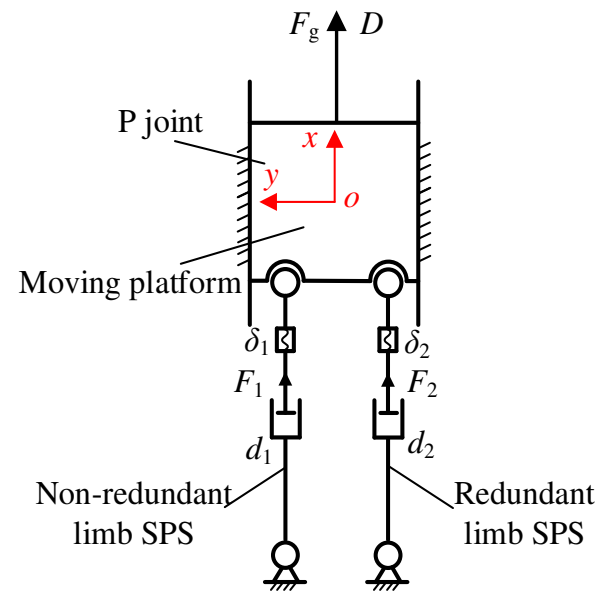

Fig. 4. The 2SPS+P active overconstrained mechanism

It is assumed that the stiffness of the moving platform is far greater than the stiffness of limbs, the main deformation source is limb axial deformation. The axial deformation of the non-redundant limb and the redundant limb are $\delta_{1}$ and $\delta_{2}$, respectively. The output displacements are $d_{1}$ and $d_{2}$, respectively, the displacement of the moving platform is $D$. The compatibility relationship of displacement can be expressed as:

$$
d_{1}+\delta_{1}=d_{2}+\delta_{2}=D
$$

The relationship between the axial elastic deformation of the limbs and the actuated force can be described as:

$$
\left(\begin{array}{l}
F_{1} \\
F_{2}
\end{array}\right)=\left(\begin{array}{ll}
k_{1} & 0 \\
0 & k_{2}
\end{array}\right)\left(\begin{array}{l}
\delta_{1} \\
\delta_{2}
\end{array}\right)
$$

where $k_{1}$ and $k_{2}$ represent the axial stiffness of the non-redundant limb and the redundant limb, respectively. Parameters $F_{1}$ and $F_{2}$ represent the actuated force of the non-redundant limb and the redundant limb, respectively.

The force balance equation established on the moving platform can be obtained:

$$
\boldsymbol{G F}=F_{g}
$$

where $\boldsymbol{G}=\left(\begin{array}{ll}1 & 1\end{array}\right), \boldsymbol{F}=\left(F_{1} F_{2}\right)^{\mathrm{T}}$ and $F_{g}$ represents generalized external force.

In full position control mode, displacements $d_{1}$ and $d_{2}$ are actively provided. Hence, the displacement compatibility described in Eq. (15) can be transformed into:

$$
\left(\begin{array}{l}
\delta_{1} \\
\delta_{2}
\end{array}\right)=\boldsymbol{G}^{\mathrm{T}} D-\left(\begin{array}{l}
d_{1} \\
d_{2}
\end{array}\right)
$$

By combining Eqs. (16) (18), the actuated force of the non-redundant limb and the redundant limb in full 
position control mode can be obtained as:

$$
\boldsymbol{F}=\boldsymbol{G}_{\boldsymbol{W}}^{+}\left(F_{g}-\boldsymbol{G} \boldsymbol{W}^{-1}\left(\begin{array}{l}
d_{1} \\
d_{2}
\end{array}\right)\right)+\boldsymbol{W}^{-1}\left(\begin{array}{l}
d_{1} \\
d_{2}
\end{array}\right)=\left(k_{1}+k_{2}\right)^{-1}\left(\begin{array}{c}
F_{g} k_{1}+k_{1} k_{2}\left(d_{1}-d_{2}\right) \\
F_{g} k_{2}+k_{1} k_{2}\left(d_{2}-d_{1}\right)
\end{array}\right)
$$

where $\boldsymbol{W}=\operatorname{diag}^{-1}\left(k_{1}, k_{2}\right), \boldsymbol{G}_{\boldsymbol{W}}^{+}=\boldsymbol{W}^{-1} \boldsymbol{G}^{\mathrm{T}}\left(\boldsymbol{G} \boldsymbol{W}^{-1} \boldsymbol{G}^{\mathrm{T}}\right)^{-1}$ represents the $W^{-}$weighted generalized inverse of the force mapping matrix $\boldsymbol{G}$.

When $d_{1}=d_{2}=d$, Eq. (19) is simplified to:

$$
\left(\begin{array}{l}
F_{1} \\
F_{2}
\end{array}\right)=\frac{F_{g}}{k_{1}+k_{2}}\left(\begin{array}{l}
k_{1} \\
k_{2}
\end{array}\right)
$$

By combining Eqs. (15), (16) and (20), the following expression can be obtained:

$$
F_{g}=\left(k_{1}+k_{2}\right)(D-d)
$$

The detailed deduction process of the above part can be resorted to reference [41].

According to Eq. (21), when the output displacement of the non-redundant limb and the redundant limb are equal, the distribution of the actuated force of two limbs is consistent with the passive overconstrained mechanism. In such cases, the equivalent stiffness of the mechanism is equal to $K_{\mathrm{e}}=k_{1}+k_{2}$.

According to Eq. (19), if the output displacements of the non-redundant limb and the redundant limb are inconsistent, the actuated forces of two limbs still exist in Eq. (20). Furthermore, there is a part of the actuated force equal to $k_{1} k_{2}\left(k_{1}+k_{2}\right)^{-1}\left(\begin{array}{l}d_{1}-d_{2} \\ d_{2}-d_{1}\end{array}\right)$, which means that the sum of the actuated forces of these two limbs is zero. This part called an internal force, i.e., the force that does not resist external forces but balances each other within the mechanism. The greater the difference between the output displacements of the two limbs, the greater the internal force which is produced by the two actuated limbs. Therefore, the output displacements of the two limbs must be consistent to ensure the system's compatibility motion without the internal force.

\subsubsection{7-SPS active overconstrained mechanism}

The 7-SPS active overconstrained mechanism is shown in Fig. 5. The main difference from the 7-SS passive overconstrained mechanism is that the P joints along the axis of each limb are added to seven SS limbs, and they are defined as actuated joints. Each of the seven SPS limbs has a local DOF allowing the rotation around the P joint axis, which does not enforce constraints on moving platform. Therefore, the moving platform has six DOFs, while the mechanism has seven actuations. Hence, the 7-SPS mechanism belongs to active overconstrained mechanism. In Fig. 5, limbs 1-6 are non-redundant limbs, while limb 7 is redundant limb. 


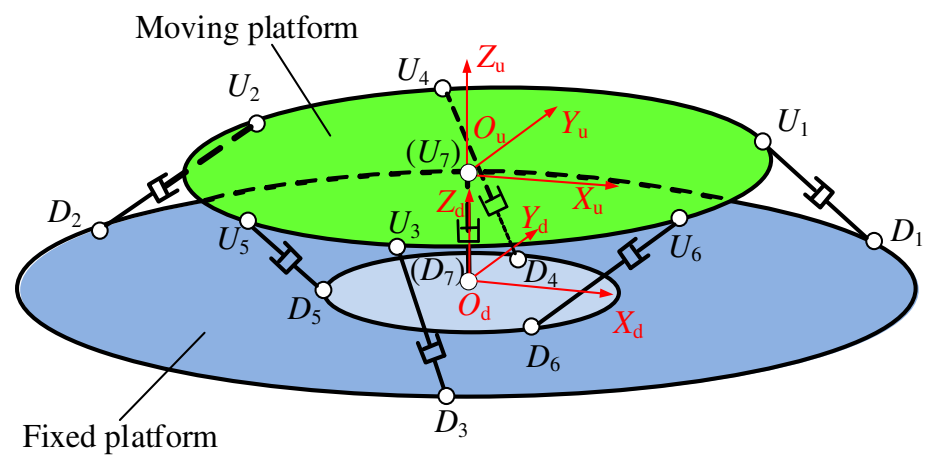

Fig. 5. The 7-SPS active overconstrained mechanism

Under assumption of negligible limb gravity effects, each limb only provides one actuated force vector along the limb axis towards the moving platform. The force balance equation for the moving platform is:

$$
\boldsymbol{\$}_{F g}=F_{1} \hat{\boldsymbol{S}}_{1}+F_{2} \hat{\boldsymbol{S}}_{2}+\cdots+F_{7} \hat{\boldsymbol{S}}_{7}=\boldsymbol{G}_{F}^{F g} \boldsymbol{F}
$$

where $S_{F g}=\left(\begin{array}{lllllll}F_{x} & F_{y} & F_{z} & M_{x} & M_{y} & M_{z}\end{array}\right)^{\mathrm{T}}$ represents the six-dimensional external force received by the moving platform. $\quad \boldsymbol{G}_{F}^{F g}=\left(\begin{array}{llll}\hat{\boldsymbol{S}}_{1} & \hat{\boldsymbol{S}}_{2} & \mathrm{~L} & \hat{\boldsymbol{S}}_{7}\end{array}\right), \quad \hat{\boldsymbol{S}}_{i}(i=1,2, \mathrm{~L}, 7)$ represents the unit wrench of the actuated force vector $\boldsymbol{S}_{i}$. $\boldsymbol{F}=\left(\begin{array}{llll}F_{1} & F_{2} & \mathrm{~L} & F_{7}\end{array}\right)^{\mathrm{T}}, \quad F_{i}$ represents the magnitude of the actuated force vector $\boldsymbol{S}_{i}$.

Assuming superior stiffness of the moving platform compared to the stiffness of the seven limbs, the moving platform is considered as a rigid body, and the main deformation source is limb axial deformation. Parameter $\Delta \boldsymbol{l}$ is the sum of the axial elastic deformations $\delta_{i}$ generated by each limb and the actuation output displacement $d_{i}$, which can be written by employing the six-dimensional displacement $\boldsymbol{D}$ generated by the moving platform under the external force:

$$
\Delta \boldsymbol{l}=\left(\begin{array}{llll}
\hat{\boldsymbol{S}}_{1} & \hat{\boldsymbol{S}}_{2} & \mathrm{~L} & \hat{\boldsymbol{S}}_{7}
\end{array}\right)^{\mathrm{T}} \boldsymbol{D}=\left(\boldsymbol{G}_{F}^{F g}\right)^{\mathrm{T}} \boldsymbol{D}
$$

where $\Delta \boldsymbol{l}=\left[\begin{array}{llll}\Delta l_{1} & \Delta l_{2} & \cdots & \Delta l_{7}\end{array}\right]^{\mathrm{T}}, \Delta l_{i}=\delta_{i}+d_{i}, i=1,2, \mathrm{~L} 7, \boldsymbol{D}=\left(\begin{array}{llllll}\delta_{x} & \delta_{y} & \delta_{z} & \varphi_{x} & \varphi_{y} & \varphi_{z}\end{array}\right)^{\mathrm{T}}$.

If $\boldsymbol{G}_{1}=\left(\begin{array}{llll}\hat{\boldsymbol{S}}_{1} & \hat{\boldsymbol{S}}_{2} & \mathrm{~L} & \hat{\boldsymbol{S}}_{6}\end{array}\right), \boldsymbol{G}_{2}=\hat{\boldsymbol{S}}_{7}$, then $\boldsymbol{G}_{F}^{F g}=\left(\begin{array}{ll}\boldsymbol{G}_{1} & \boldsymbol{G}_{2}\end{array}\right)$. Thus, Eq. (23) can be divided into:

$$
\left\{\begin{array}{l}
{\left[\begin{array}{llll}
\Delta l_{1} & \Delta l_{2} & \cdots & \Delta l_{6}
\end{array}\right]^{\mathrm{T}}=\boldsymbol{G}_{1}^{\mathrm{T}} \boldsymbol{D}} \\
\Delta l_{7}=\boldsymbol{G}_{2}^{\mathrm{T}} \boldsymbol{D}
\end{array}\right.
$$

If $\boldsymbol{G}_{1}$ is invertible, then the following expression can be written:

$$
\boldsymbol{D}=\left(\boldsymbol{G}_{1}^{\mathrm{T}}\right)^{-1}\left[\begin{array}{llll}
\Delta l_{1} & \Delta l_{2} & \cdots & \Delta l_{6}
\end{array}\right]^{\mathrm{T}}
$$


By substituting Eq. (25) into Eq. (24):

$$
\Delta l_{7}=\left(\boldsymbol{G}_{1}^{-1} \boldsymbol{G}_{2}\right)^{\mathrm{T}}\left[\begin{array}{llll}
\Delta l_{1} & \Delta l_{2} & \cdots & \Delta l_{6}
\end{array}\right]^{\mathrm{T}}
$$

The relationship between the axial elastic deformation of the limb and the actuated force can be expressed as follows:

$$
\boldsymbol{F}=\boldsymbol{K} \boldsymbol{\delta}=\boldsymbol{K}(\Delta \boldsymbol{l}-\boldsymbol{d})
$$

where $\boldsymbol{K}=\operatorname{diag}\left(k_{1} \quad k_{2} \quad \mathrm{~L} \quad k_{7}\right), \quad \boldsymbol{\delta}=\left[\begin{array}{llll}\delta_{1} & \delta_{2} & \cdots & \delta_{7}\end{array}\right]^{\mathrm{T}}, \boldsymbol{d}=\left[\begin{array}{llll}d_{1} & d_{2} & \cdots & d_{7}\end{array}\right]^{\mathrm{T}}$.

By combining Eqs. (26) and (27), yields:

$$
k_{7}^{-1} F_{7}=\boldsymbol{B}^{\mathrm{T}} \boldsymbol{K}_{1}^{-1} \boldsymbol{F}_{1}+\boldsymbol{B}^{\mathrm{T}} \boldsymbol{d}_{1}-d_{7}
$$

where $\boldsymbol{K}_{1}=\operatorname{diag}\left(k_{1} \quad k_{2} \quad \mathrm{~L} \quad k_{6}\right), \boldsymbol{F}_{1}=\left(\begin{array}{lllll}F_{1} & F_{2} & \mathrm{~L} & F_{6}\end{array}\right)^{\mathrm{T}}, \boldsymbol{d}_{1}=\left[\begin{array}{llll}d_{1} & d_{2} & \cdots & d_{6}\end{array}\right]^{\mathrm{T}}$, represent the limbs stiffness, actuated forces and output displacements of the six non-redundant limbs, respectively. Parameter $B$ is equal to $\boldsymbol{B}=\boldsymbol{G}_{1}^{-1} \boldsymbol{G}_{2}$.

Eq. (28) can be transformed into:

$$
\left[\begin{array}{cc}
\boldsymbol{B}^{\mathrm{T}} \boldsymbol{K}_{1}^{-1} & -k_{7}^{-1}
\end{array}\right] \boldsymbol{F}=d_{7}-\boldsymbol{B}^{\mathrm{T}} \boldsymbol{d}_{1}
$$

By combining Eq. (22) and Eq. (29), the following expression is obtained:

$$
\left[\begin{array}{ll}
\boldsymbol{S}_{F g} & d_{7}-\boldsymbol{B}^{\mathrm{T}} \boldsymbol{d}_{1}
\end{array}\right]^{\mathrm{T}}=\left[\begin{array}{cc}
\boldsymbol{G}_{1} & \boldsymbol{G}_{2} \\
\boldsymbol{B}^{\mathrm{T}} \boldsymbol{K}_{1}^{-1} & -k_{7}^{-1}
\end{array}\right] \boldsymbol{F}
$$

According to Eq. (30), the actuated forces of the non-redundant and redundant limbs can be obtained as follows:

$$
\begin{gathered}
\boldsymbol{F}=\boldsymbol{A}\left[\begin{array}{ll}
\boldsymbol{S}_{F g} & d_{7}-\boldsymbol{B}^{\mathrm{T}} \boldsymbol{d}_{1}
\end{array}\right]^{\mathrm{T}} \\
\text { where } \boldsymbol{A}=\left[\begin{array}{cc}
\boldsymbol{G}_{1} & \boldsymbol{G}_{2} \\
\boldsymbol{B}^{\mathrm{T}} \boldsymbol{K}_{1}^{-1} & -k_{7}^{-1}
\end{array}\right]^{-1}=\left[\begin{array}{cc}
\boldsymbol{A}_{1} & \boldsymbol{A}_{2} \\
\boldsymbol{A}_{3} & \boldsymbol{A}_{4}
\end{array}\right]=\left[\begin{array}{cc}
\left(\boldsymbol{G}_{1}+\boldsymbol{G}_{2} k_{7} \boldsymbol{B}^{\mathrm{T}} \boldsymbol{K}_{1}^{-1}\right)^{-1} & \boldsymbol{B}\left(k_{7}^{-1}+\boldsymbol{B}^{\mathrm{T}} \boldsymbol{K}_{1}^{-1} \boldsymbol{B}\right)^{-1} \\
k_{7} \boldsymbol{B}^{\mathrm{T}} \boldsymbol{K}_{1}^{-1}\left(\boldsymbol{G}_{1}+\boldsymbol{G}_{2} k_{7} \boldsymbol{B}^{\mathrm{T}} \boldsymbol{K}_{1}^{-1}\right)^{-1} & -\left(k_{7}^{-1}+\boldsymbol{B}^{\mathrm{T}} \boldsymbol{K}_{1}^{-1} \boldsymbol{B}\right)^{-1}
\end{array}\right] .
\end{gathered}
$$

Eq. (31) can be transformed into:

$$
\boldsymbol{F}=\left[\begin{array}{ll}
\boldsymbol{A}_{1} & \boldsymbol{A}_{3}
\end{array}\right]^{\mathrm{T}} \boldsymbol{S}_{F g}+\left[\begin{array}{ll}
\boldsymbol{A}_{2} & \boldsymbol{A}_{4}
\end{array}\right]^{\mathrm{T}}\left(d_{7}-\boldsymbol{B}^{\mathrm{T}} \boldsymbol{d}_{1}\right)
$$

Eq. (32) is further simplified as:

$$
\boldsymbol{F}=\left(\boldsymbol{G}_{F}^{F g}\right)_{W}^{+} \boldsymbol{S}_{F g}+\left[\begin{array}{ll}
\boldsymbol{A}_{2} & \boldsymbol{A}_{4}
\end{array}\right]^{\mathrm{T}}\left(d_{7}-\boldsymbol{B}^{\mathrm{T}} \boldsymbol{d}_{1}\right)
$$


where $\left(\boldsymbol{G}_{F}^{F g}\right)_{W}^{+}$represents the $\boldsymbol{W}$-weighted generalized inverse of the force mapping matrix $\boldsymbol{G}_{F}^{F g}$, $\boldsymbol{W}=\operatorname{diag}^{-1}\left(\begin{array}{llll}k_{1} & k_{2} & \mathrm{~L} & k_{7}\end{array}\right),\left(\boldsymbol{G}_{F}^{F g}\right)_{W}^{+}=\boldsymbol{W}^{-1}\left(\boldsymbol{G}_{F}^{F g}\right)^{\mathrm{T}}\left(\boldsymbol{G}_{F}^{F g} \boldsymbol{W}^{-1}\left(\boldsymbol{G}_{F}^{F g}\right)^{\mathrm{T}}\right)^{-1}$.

According to Eq. (32), in the full position control mode, the actuated force of the active overconstrained mechanism 7-SPS can be actively adjusted via actuation output displacement. Part of the actuated force is employed to balance the external force, while the other part is used to coordinate internal force due to the difference in actuation output displacements. When the output displacement of the redundant limb is equal to $d_{7}=\boldsymbol{B}^{\mathrm{T}} \boldsymbol{d}_{1}$, the internal force is equal to $\left[\begin{array}{ll}\boldsymbol{A}_{2} & \boldsymbol{A}_{4}\end{array}\right]^{\mathrm{T}}\left(d_{7}-\boldsymbol{B}^{\mathrm{T}} \boldsymbol{d}_{1}\right)=0$. Distribution of the actuated force of each limb is consistent with the 7-SS passive overconstrained mechanism, while the equivalent stiffness of the mechanism is equal to $\boldsymbol{K}_{e}=\boldsymbol{G}_{F}^{F g} \boldsymbol{K}\left(\boldsymbol{G}_{F}^{F g}\right)^{\mathrm{T}}$.

\subsection{Passive-input overconstrained mechanisms}

Taking 2SPS+P and 7-SPS passive-input overconstrained mechanisms as examples, the equivalent stiffness and distribution of actuated force of each limb are solved.

\subsubsection{SPS+P passive-input overconstrained mechanism}

The 2SPS+P active overconstrained mechanism is shown in Fig. 4. If spring actuation is employed, the mechanism becomes a passive-input overconstrained mechanism, as shown in Fig. 6. Unlike the active overconstrained mechanism, the spring represents a passive-input, and its actuated mode cannot be actively controlled.

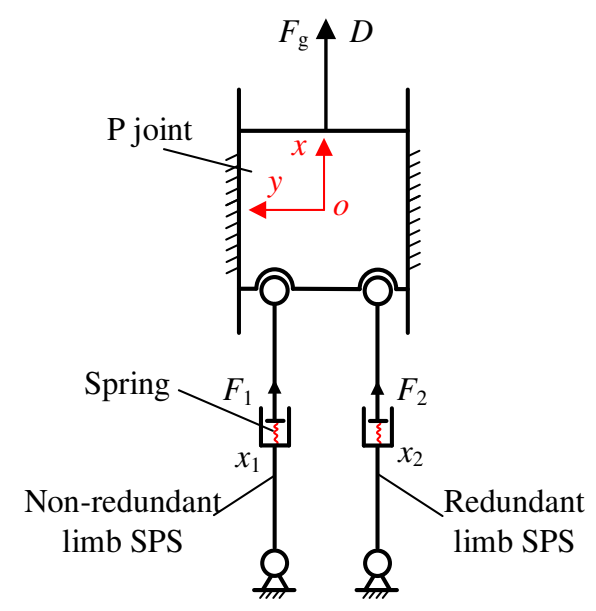

Fig.6. The 2SPS+P passive-input overconstrained mechanism 
Force analysis of the 2SPS+P passive-input overconstrained mechanism is carried out, the main deformation source is passive spring deformation, with parameters $k_{1}$ and $k_{2}$ representing the non-redundant and redundant limb stiffnesses. The output displacement of the actuation, i.e., the deformation of the spring, is denoted as $x_{1}$ and $x_{2}$, respectively. If initial compression of the spring is equal to $x_{10}$ and $x_{20}$, and the displacement of the moving platform is $D$, then the displacement compatibility relationship of the mechanism can be expressed as:

$$
x_{1}=x_{2}=D
$$

If $x_{1}=x_{2}=D=x$, the mechanism represents a single-DOF mechanism. There is a single independent generalized coordinate, and $x$ is selected as the generalized coordinate. Dynamic equation is established according to the second Lagrange equation, which can be expressed as:

$$
\frac{\mathrm{d}}{\mathrm{d} t} \frac{\partial E_{v}}{\partial \&}-\frac{\partial E_{v}}{\partial x}+\frac{\partial E_{P}}{\partial x}=F_{g}
$$

where $E_{v}$ and $E_{P}$ represent kinetic and potential energy of the mechanism, respectively, and $F_{g}$ represents the generalized external force.

During motion, the moving platform and the connecting links move in translation. Total kinetic and potential energy of the mechanism can be expressed as follows:

$$
\begin{gathered}
E_{v}=\frac{1}{2}\left(M_{D}+M_{1}+M_{2}\right) \& \\
E_{P}=\frac{1}{2} k_{1}\left(x_{10}-x\right)^{2}+\frac{1}{2} k_{2}\left(x_{20}-x\right)^{2}
\end{gathered}
$$

where $M_{D}, M_{1}$ and $M_{2}$ represent masses of the moving platform and the two limb connecting links, respectively.

By substituting Eqs. (36) and (37) into Eq. (35), dynamic equation can be derived as:

$$
M K_{e} x+K_{e} x_{0}=F_{g}
$$

where $M=M_{D}+M_{1}+M_{2}$, $\frac{F_{g}-F_{1}-F_{2}}{M_{D}}, K_{\mathrm{e}}=k_{1}+k_{2}, x_{0}=\frac{k_{1} x_{10}+k_{2} x_{20}}{K_{e}}$ 。

The relationship between the axial displacements of the limbs and actuated forces can be obtained:

$$
\left(\begin{array}{l}
F_{1} \\
F_{2}
\end{array}\right)=\left(\begin{array}{l}
k_{1} x_{10} \\
k_{2} x_{20}
\end{array}\right)-\left(\begin{array}{cc}
k_{1} & 0 \\
0 & k_{2}
\end{array}\right)\left(\begin{array}{l}
x \\
x
\end{array}\right)
$$


By substituting Eq. (39) into Eq. (38):

$$
x=-\frac{F_{g}}{K_{e}}+\frac{M_{D}-1}{M_{D}-M} x_{0}
$$

The equivalent stiffness of the $2 \mathrm{SPS}+\mathrm{P}$ passive-input overconstrained mechanism is equal to $K_{\mathrm{e}}=k_{1}+k_{2}$. When the passive spring stiffness is provided, the displacement of the moving platform can be obtained according to Eq. (40). Then, actuated force of the non-redundant and redundant limbs can be calculated according to Eq. (39).

\subsubsection{7-SPS passive-input overconstrained mechanism}

The 7-SPS active overconstrained mechanism is shown in Fig. 5. If spring actuations are employed (Fig. 7), the mechanism becomes passive-input overconstrained mechanism.

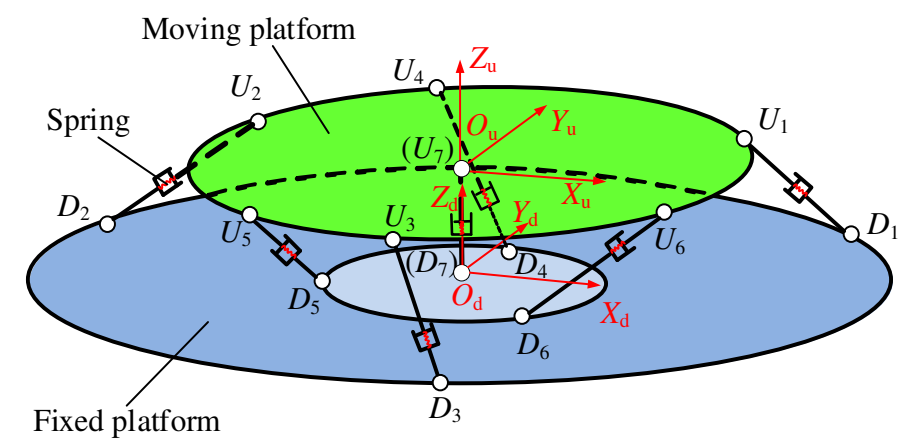

Fig.7 The 7-SPS passive-input overconstrained mechanism

The 7-SPS pssive-input overconstrained mechanism has six DOFs. ${ }^{O \mathrm{~d}} \boldsymbol{P}_{O \mathrm{u}}$ and ${ }^{O \mathrm{~d}} \boldsymbol{R}_{O \mathrm{u}}$ are used to describe both position and the orientation of the moving platform, respectively. The position vector of the $\mathrm{S}$ joint on the fixed platform is expressed as:

$$
{ }^{O \mathrm{~d}} \boldsymbol{D}_{i}=\left(\begin{array}{lll}
{ }^{O \mathrm{~d}}{ }^{x_{D i}} & { }^{O \mathrm{~d}} y_{D i} & { }^{O \mathrm{~d}} z_{D i}
\end{array}\right)^{\mathrm{T}}, i=1,2, \ldots 7
$$

where ${ }^{O \mathrm{~d}} x_{D i},{ }^{O \mathrm{~d}} y_{D i}$ and ${ }^{O \mathrm{~d}} z_{D i}$ are obtained according to the circumferential distribution of the $\mathrm{S}$ joint on the fixed platform.

The position vector of the $\mathrm{S}$ joint on the moving platform is expressed as:

$$
{ }^{O \mathrm{u}} \boldsymbol{U}_{i}=\left(\begin{array}{lll}
{ }^{O \mathrm{u}} x_{U i} & { }^{O \mathrm{u}} y_{U i} & { }^{O \mathrm{u}} z_{U i}
\end{array}\right)^{\mathrm{T}}, i=1,2, \ldots 7
$$

where ${ }^{O \mathrm{u}} x_{U i},{ }^{O \mathrm{u}} y_{U i}$ and ${ }^{O \mathrm{u}} z_{U i}$ are obtained according to the circumferential distribution of the $\mathrm{S}$ joint on the moving platform. 
For each limb, the closed-loop equation is satisfied:

$$
d_{i}\left(\hat{\boldsymbol{S}}_{i}\right)_{1: 3,1}={ }^{O \mathrm{~d}} \boldsymbol{P}_{O \mathrm{u}}+{ }^{O \mathrm{~d}} \boldsymbol{U}_{i}-{ }^{O \mathrm{~d}} \boldsymbol{D}_{i}={ }^{O \mathrm{~d}} \boldsymbol{P}_{O \mathrm{u}}+{ }^{O \mathrm{~d}} \boldsymbol{R}_{O \mathrm{u}}{ }^{\mathrm{ou}} \boldsymbol{U}_{i}-{ }^{O \mathrm{~d}} \boldsymbol{D}_{i}
$$

where $\hat{\boldsymbol{S}}_{i}$ is the unit wrench of the actuated force wrench and $\left(\hat{\boldsymbol{S}}_{i}\right)_{1: 3,1}$ represents the first three lines of $\hat{\boldsymbol{S}}_{i}$ In order to obtain the output displacement $d_{i}$ of actuation and eliminate $\left(\hat{\boldsymbol{S}}_{i}\right)_{1: 3,1}$, two sides of Eq. (43) are dot-multiplied by themselves:

$$
d_{i}^{2}=\left[{ }^{O \mathrm{~d}} \boldsymbol{P}_{O \mathrm{u}}+{ }^{O \mathrm{~d}} \boldsymbol{R}_{O \mathrm{u}}{ }^{\mathrm{Ou}} \boldsymbol{U}_{i}-{ }^{O \mathrm{~d}} \boldsymbol{D}_{i}\right]^{\mathrm{T}}\left[{ }^{O \mathrm{~d}} \boldsymbol{P}_{O \mathrm{u}}+{ }^{O \mathrm{~d}} \boldsymbol{R}_{O \mathrm{u}}{ }^{O \mathrm{u}} \boldsymbol{U}_{i}-{ }^{O \mathrm{~d}} \boldsymbol{D}_{i}\right]
$$

Therefore, the output displacement can be derived as follows:

$$
d_{i}=\left[\left[{ }^{O \mathrm{~d}} \boldsymbol{P}_{O \mathrm{u}}+{ }^{O \mathrm{~d}} \boldsymbol{R}_{O \mathrm{u}}{ }^{\mathrm{Ou}} \boldsymbol{U}_{i}-{ }^{O \mathrm{~d}} \boldsymbol{D}_{i}\right]^{\mathrm{T}}\left[{ }^{O \mathrm{~d}} \boldsymbol{P}_{O \mathrm{u}}+{ }^{O \mathrm{~d}} \boldsymbol{R}_{O \mathrm{u}}{ }^{O \mathrm{u}} \boldsymbol{U}_{i}-{ }^{O \mathrm{~d}} \boldsymbol{D}_{i}\right]\right]^{1 / 2}
$$

By employing the same equivalent stiffness solution of the 7-SS passive overconstrained mechanism, the equivalent stiffness of the 7-SPS passive-input overconstrained mechanism can be expressed as:

$$
\boldsymbol{K}_{e}=\boldsymbol{G}_{F}^{F g} \boldsymbol{K}\left(\boldsymbol{G}_{F}^{F g}\right)^{\mathrm{T}}
$$

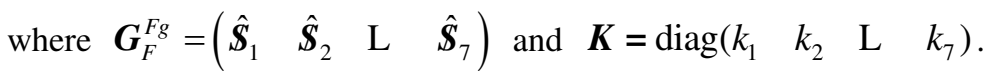

Since configuration of the mechanism varies with time, the mapping matrix $\boldsymbol{G}_{F}^{F g}$ is also altered, that is, $\boldsymbol{K}_{e}$ changes in real time. According to Eq. (43) yields:

$$
\left(\hat{\boldsymbol{S}}_{i}\right)_{1: 3,1}=\left({ }^{O \mathrm{~d}} \boldsymbol{P}_{O \mathrm{u}}+{ }^{O \mathrm{~d}} \boldsymbol{R}_{O \mathrm{u}}{ }^{O \mathrm{u}} \boldsymbol{U}_{i}-{ }^{O \mathrm{~d}} \boldsymbol{D}_{i}\right) / d_{i}
$$

The six-dimensional displacement, $\boldsymbol{D}$, of the moving platform under the action of an external force can be calculated as follows:

$$
\boldsymbol{D}=\int\left(\boldsymbol{K}_{e}^{-1} \boldsymbol{F}_{g}\right) d t=\int\left[\left(\boldsymbol{G}_{F}^{F g} \boldsymbol{K}\left(\boldsymbol{G}_{F}^{F g}\right)^{\mathrm{T}}\right)^{-1} \boldsymbol{F}_{g}\right] d t
$$

Axial displacement and actuated force of each limb must satisfy the following expression:

$$
\boldsymbol{F}=\boldsymbol{F}_{0}-\boldsymbol{K} \boldsymbol{d}
$$

where $\boldsymbol{F}=\left(\begin{array}{lllllllll}F_{1} & F_{2} & \mathrm{~L} & F_{7}\end{array}\right)^{\mathrm{T}}$ represents the actuated force of each limb, $\boldsymbol{F}_{0}=\left(\begin{array}{llll}F_{10} & F_{20} & \mathrm{~L} & F_{70}\end{array}\right)^{\mathrm{T}}$ represents the preload of each limb spring, and $\boldsymbol{d}=\left(\begin{array}{llll}d_{1} & d_{2} & \mathrm{~L} & d_{7}\end{array}\right)^{\mathrm{T}}$ represents the output displacement of each limb. 
Combining the Eq. (43) with Eqs. (48) and (49), the output displacement of the moving platform, output displacement of actuation and actuated force of actuation can be obtained.

\subsubsection{Simulation verification}

The 7-SPS passive-input overconstrained mechanism is considered as an example for the simulation analysis. Initial mechanism parameters are consistent with the 7-SS passive overconstrained mechanism. Since the mechanism is actuated via springs, limb deformations are not considered, the rigid body model of the mechanism is employed as Fig. 8. Spring stiffness of limbs 1-7 is taken as $\boldsymbol{K}=\left[\begin{array}{lllllll}6.3 & 5.3 & 1.3 & 6.3 & 35.3 & 7.3 & 16\end{array}\right]^{\mathrm{T}} \times 10^{3} \mathrm{~N} / \mathrm{m}$. The spring preload is set to $100 \mathrm{~N}$, and an external force $\$_{F}=[-10 \mathrm{~N}-25 \mathrm{~N}-20 \mathrm{~N} 0 \mathrm{~N} \cdot \mathrm{m} 0 \mathrm{~N} \cdot \mathrm{m} 35 \mathrm{~N} \cdot \mathrm{m}]^{\mathrm{T}}$ is applied to the center of the moving platform. Comparison between theoretical and simulation values of actuated forces is shown in Fig. 9.

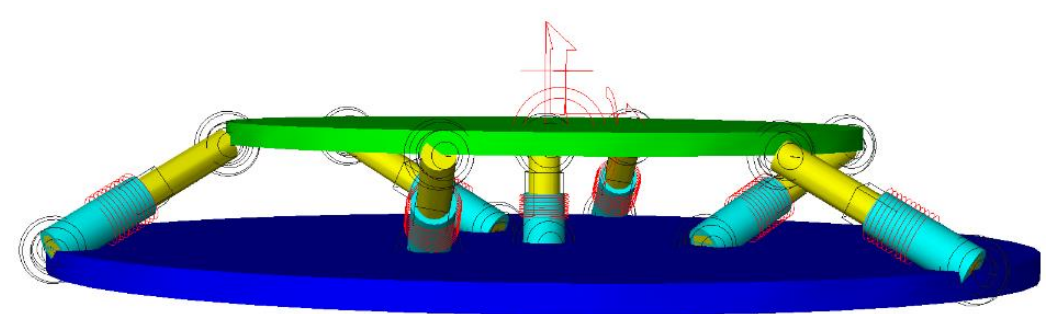

Fig. 8. Simulation model of the 7-SPS passive-input overconstrained mechanism

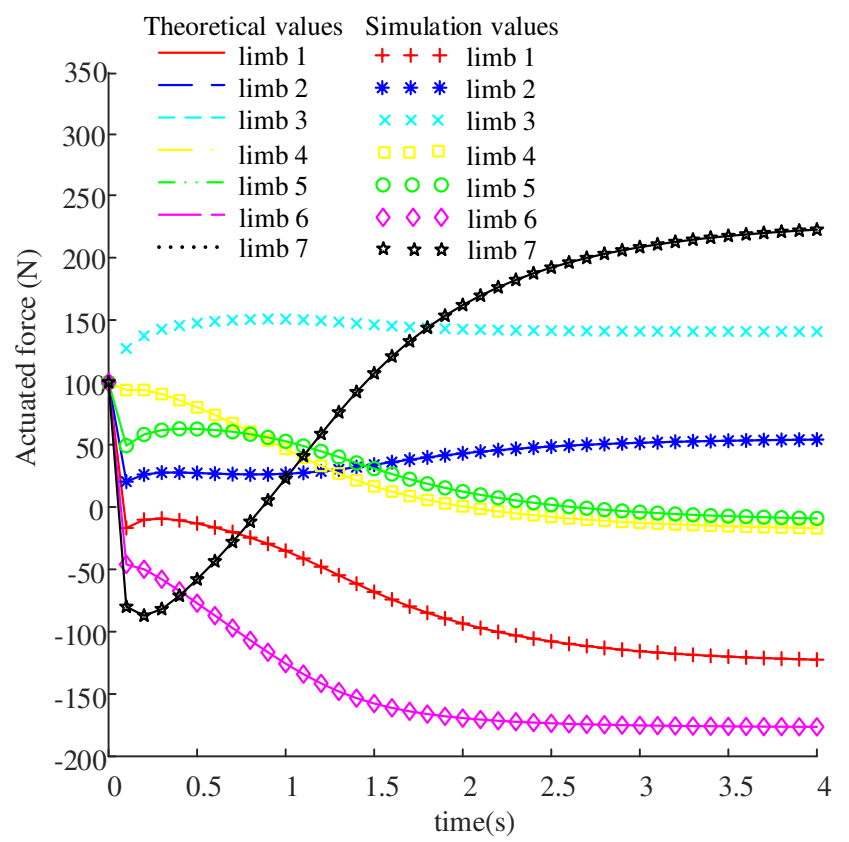

Fig.9. Comparison between theoretical and simulation values of the actuated force

According to Fig. 9, the theoretical and simulation values of actuated forces are correlating perfectly. This is because the parameters of the established simulation model are completely consistent with the theoretical model. 
Parameters such as gravity, joint shape and friction are not considered. Thus, each component represents a rigid body without errors. It can be concluded that force analysis of the passive-input overconstrained mechanism based on the equivalent stiffness method is verified.

\section{Adaptability of the equivalent stiffness to three different types of overconstrained mechanisms}

Main goal of passive overconstrained, active overconstrained and passive-input overconstrained mechanisms force analysis is to obtain the equivalent stiffness based on the equivalent stiffness method. According to Section 2, the equivalent stiffness of three types of overconstrained mechanisms can be uniformly expressed as:

$$
\boldsymbol{K}_{e}=\boldsymbol{G}_{f}^{F} \boldsymbol{K}\left(\boldsymbol{G}_{f}^{F}\right)^{\mathrm{T}}
$$

For passive overconstrained mechanism, by considering the axial elastic deformation of limbs, the mechanism force balance equation should first be established. Then, virtual work principle should be combined to obtain deformation compatibility equation of the mechanism. According to the relationship between the axial stiffness and limb deformation, the relationship between the external force and the output displacement of the moving platform can be established. Furthermore, the equivalent stiffness matrix of the passive overconstrained mechanism can be obtained.

For active overconstrained mechanism in the position control mode, the output displacement of actuation is provided by the inverse kinematics solution of the mechanism. This is done by combining the force balance and displacement compatibility equations to establish the analytical relationship between the magnitudes of actuated wrenches and the external force with the limb stiffnesses to obtain the equivalent stiffness matrix. The equivalent stiffness of the passive-input overconstrained mechanism is comparable to the passive overconstrained mechanism. For a single-DOF passive-input overconstrained mechanism, its equivalent stiffness is constant. For multi-DOF passive-input overconstrained mechanism $(M>1)$, its equivalent stiffness is altered in real time.

The force analysis method based on the idea of equivalent stiffness is suitable for all three types of overconstrained mechanisms. General expression can be written as:

$$
\boldsymbol{F}=\boldsymbol{F}_{0} \pm \boldsymbol{K}\left(\boldsymbol{G}_{f}^{F}\right)^{\mathrm{T}} \boldsymbol{D}\left(\boldsymbol{K}_{e}\right)+\boldsymbol{F}_{i n}
$$

where $\boldsymbol{F}$ represents the restraint or actuated force/couples, $\boldsymbol{F}_{0}$ represents the initial preload of actuation, $\boldsymbol{K}=\operatorname{diag}\left(k_{1} \quad k_{2} \quad \mathrm{~L} \quad k_{i}\right)$ represents the vector matrix composed of the stiffness of each limb, $\boldsymbol{G}_{f}^{F}$ represents the force mapping matrix from limbs to the moving platform, $\boldsymbol{D}\left(\boldsymbol{K}_{e}\right)$ represents the output 
displacement function of the moving platform based on the equivalent stiffness, and $\boldsymbol{F}_{\text {in }}$ represents the internal force due to the incompatibility output displacement of actuation.

According to Eqs. (50) and (51) and the equivalent stiffness, the constraint force of the passive overconstrained mechanism can be directly solved. $\boldsymbol{F}_{0}$ and $\boldsymbol{F}_{\text {in }}$ are both zero. $\boldsymbol{F}_{0}$ of the active overconstrained mechanism is zero, and the actuated force can be actively adjusted according to the output displacement of actuation. One part of the actuated force is directly solved according to the equivalent stiffness of the mechanism, while the other part is the internal force emerging from the difference in the output displacement of actuation. When the output displacements of the non-redundant limbs and the redundant limbs are compatible, the mechanism does not generate internal force. Currently, the distribution of the actuated force is consistent with the passive overconstrained mechanism. The passive-input overconstrained mechanism employs passive components (such as springs) as actuation, while the actuation mode itself cannot be actively controlled. The output displacement can be solved based on the equivalent stiffness, after which the actuated force can be solved. However, analysis process from a single-DOF mechanism to a multi-DOF mechanism is gradually becoming more complicated. For a single-DOF mechanism, the equivalent stiffness is constant. The dynamic model based on the second type of Lagrange equation is established to obtain the output displacement of the mechanism and the actuated force of each limb. For a multi-DOF mechanism, as the mechanism configuration varies with the time, its equivalent stiffness changes as well. First, the output displacement should be obtained based on the equivalent stiffness and the vector matrix integral. Then, actuated force of each limb is obtained based on mechanism kinematics inverse solution and the mechanical properties of springs.

\section{Influence mechanism of limb stiffness on the force distribution}

According to force analysis of the passive overconstrained mechanism in Section 2.1, the constraint force of the

7-SS passive overconstrained structure is equal to $\boldsymbol{f}=\boldsymbol{K}\left(\boldsymbol{G}_{f}^{F}\right)^{\mathrm{T}}\left(\boldsymbol{K}_{e}\right)^{-1} \boldsymbol{\$}_{F}$. By considering limb 1 and 6 as examples, the axial stiffness is magnified by 0.1 2 times, while stiffness of other limbs remains unchanged. Constraint force of each limb is obtained as shown in Fig. 10 and Fig. 11, respectively. 


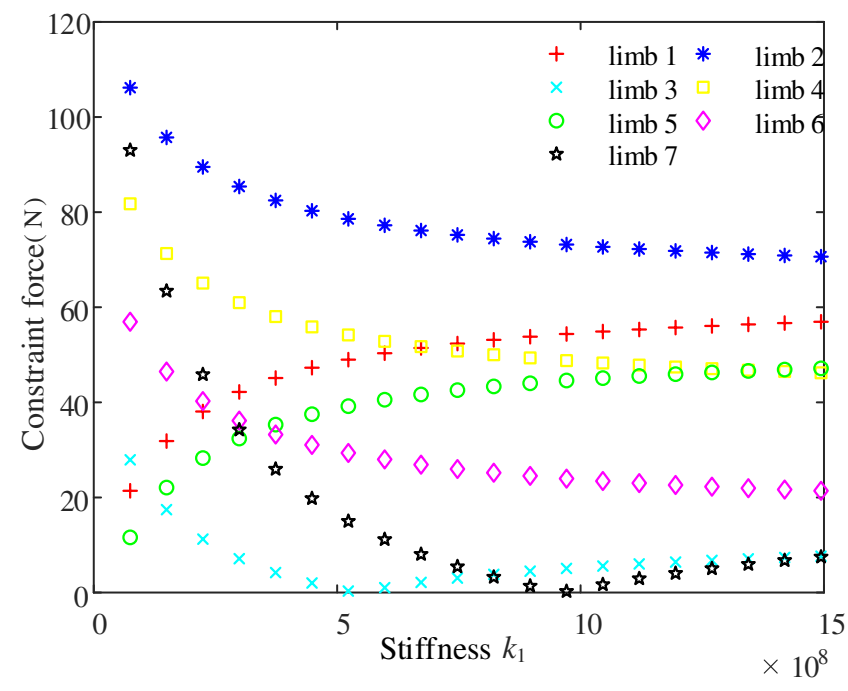

Fig. 10. Variation curve of constraint force as the function of $k_{1}$

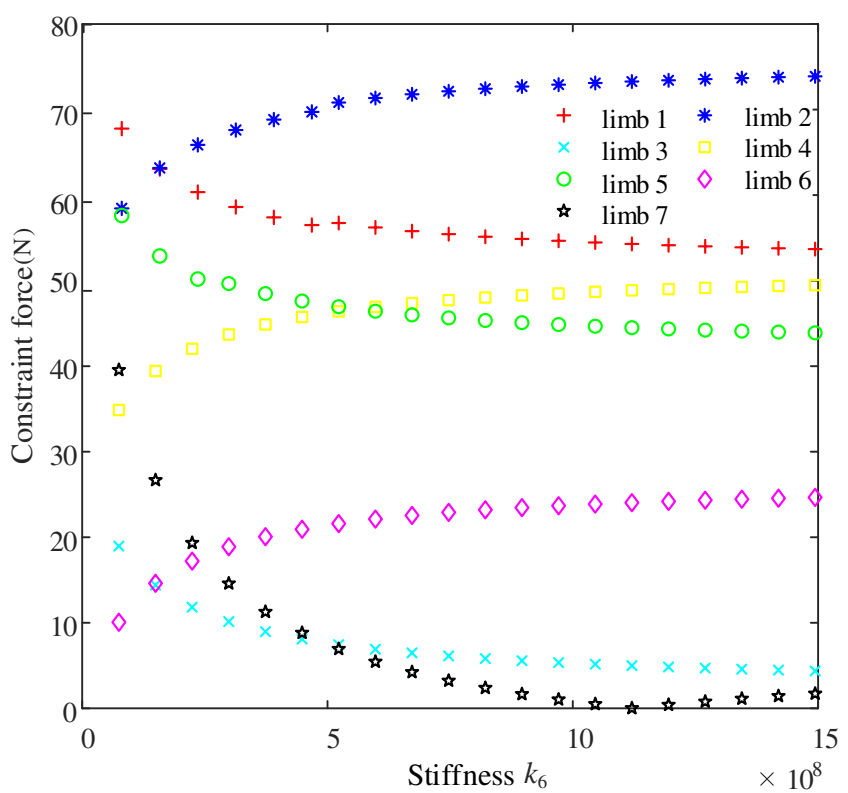

Fig. 11. Variation curve of constraint force as the function of $k_{6}$

According to Fig. 10 and Fig. 11, with an increase in the axial stiffness of limb 1 (or limb 6), an increase in the allocated constraint force is observed. The constraint forces of other limbs will increase or decrease accordingly. By analyzing other limbs in a similar manner, the same effect is observed. For the 2SS+P passive overconstrained structure, the greater the axial stiffness of the limb 1, the greater the allocated constraint force. Consequently, a decrease in the constraint force of the limb 2 is observed. Therefore, for passive overconstrained mechanism, with an increase in the limb stiffness, an increase in the constraint force follows.

According to force analysis of the active overconstrained mechanism in section 2.2 , for the full position control mode, the output displacements of the non-redundant and redundant limbs are provided by an inverse kinematics solution. Actuated forces of non-redundant and redundant limbs are determined via generalized 
external force of the system, output displacement of actuations, and the limb stiffness. When stiffness of the non-redundant and redundant limbs is equal, the limb actuated force is related to the output displacement of the limb actuation. When the output displacement of the non-redundant and redundant limbs meets the compatibility relationship, an increase in the limb stiffness will cause an increase in the actuated force. Therefore, in full position control mode of the active overconstrained parallel mechanism, only the output displacements are defined as the active quantities. On the other hand, actuated forces of non-redundant and redundant limbs are defined as the passive quantities, which cannot be directly distributed according to the optimized distribution algorithm.

According to force analysis of the passive-input overconstrained mechanism in Section 2.3, for a single-DOF passive-input mechanism (2SPS+P), its equivalent stiffness is constant. The actuated force is influenced by a generalized external force on the system, mass of the moving components and the limb stiffness. The greater the limb stiffness, the greater the actuated allocated force. For multi-DOF passive-input mechanism (7-SPS), its equivalent stiffness varies in real time. The actuated force is determined via generalized external force and the limb stiffness. When stiffness of a single limb is altered, the position and orientation of the moving platform changes, which determines the output displacement of the actuations. Consequently, the actuated force increases or decreases. In other words, limb stiffness effect of the passive-input overconstrained mechanism on the output motion in each direction is inconsistent, and it needs to be analyzed in combination with the specific mechanism.

\section{Conclusions}

(1) Force analysis method for overconstrained mechanism based on equivalent stiffness is proposed. In order to show the generality of this method for overconstrained mechanisms considering limb axial deformation, the planar mechanisms and the spatial mechanisms are analyzed respectively. Analytical force expressions including equivalent stiffness of three types of overconstrained mechanisms are obtained. Common force characteristics of three types of overconstrained mechanisms are discussed from the stiffness perspective, and various internal mechanisms between them are elaborated.

(2) The stiffness model is established by considering the compatibility of passive deformation and active displacements. The force analysis of passive, active, and passive-input overconstrained mechanisms is unified from the equivalent stiffness idea. In the future work, the authors will focus on the force analysis of overconstrained mechanism considering the axial deformation, bending deformation and torsion deformation of limbs. 


\section{Declarations:}

\section{Availability of data and materials}

The datasets used and/or analysed during the current study are available from the corresponding author on reasonable request.

\section{Competing interests}

The authors declare that they have no competing interests.

\section{Funding}

This study was financially supported by National Natural Science Foundation of China (Grant No. 52075467), National Natural Science Foundation of China (Grant No.51875495) and the Key Project of Natural Science Foundation of Hebei Province of China (Grant No. E2017203335).

\section{Authors' contributions}

Yongsheng zhao and Yundou $\mathrm{Xu}$ were responsible for the planning of the content and writing, Jinwei Guo analyzed the force principle of overconstrained mechanisms, Bo Chen performed the simulation analysis, Guoxing Zhang analyzed the research on the current situation of the manuscript, Jiantao Yao was major contributor in writing the manuscript. All authors read and approved the final manuscript.

\section{Acknowledgements}

Not applicable.

\section{References}

[1] Z. Huang, Q.C. Li. Type synthesis of symmetrical lower-mobility parallel mechanisms using the constraint-synthesis method. The International Journal of Robotics Research, 2003, 22 (1): 59-79.

[2] J.S. Dai, Z. Huang, H. Lipkin. Mobility of overconstrained parallel mechanisms. ASME Journal of Mechanical Design, 2006, 12 (8): 220-229.

[3] X.X. Chai, Q.C. Li, W. Ye. Analysis of overconstrained parallel mechanism using Grassmann-Cayley algebra. Applied Mathematical Modelling, 2017, 51(11): 643-654.

[4] Y.Q. Li, Y. Zhang, L.J. Zhang. A New Method for Type Synthesis of 2R1T and 2T1R 3-DOF Redundant Actuated Parallel Mechanisms with Closed Loop Units. Chinese Journal of Mechanical Engineering, 2020, 33(1).

[5] Y.D. Xu, J.T. Yao, Y.S. Zhao. Inverse dynamics and internal forces of the redundantly actuated parallel manipulators. Mechanism and Machine Theory, 2012, 51: 172-184.

[6] D. Liang, Y.M. Song, T. Sun, et al. Optimum design of a novel redundantly actuated parallel manipulator 
with multiple actuation modes for high kinematic and dynamic performance. Nonlinear Dynamics, 2016, 83(1): 631-658.

[7] C. Cheng, W.L. Xu, J.Z. Shang. Optimal distribution of the actuating torques for a redundantly actuated masticatory robot with two higher kinematic pairs. Nonlinear Dynamics, 2017, 79(2): 1235-1255.

[8] Y.D. Xu, Y. Chen, W.L. Liu, et al. DOF and dynamic analysis of the multi-loop coupled passive-input overconstrained deployable tetrahedral mechanisms for truss antennas. Journal of Mechanisms and Robotics, 2019, 12(1): 1-19.

[9] Z.M. Bi, B. Kang. An inverse dynamic model of over-constrained parallel kinematic machine based on Newton-Euler formulation. Journal of Dynamic Systems Measurement \& Control, 2014, 136(4): 041001.

[10] M. Wojtyra. Joint reaction forces in multibody systems with redundant constraints. Multibody System Dynamics, 2005, 14(1):23-46.

[11] J. Fraczek, M. Wojtyra. On the unique solvability of a direct dynamics problem for mechanisms with redundant constraints and coulomb friction in joints. Mechanism and Machine Theory, 2011, 46 (3): $312-334$.

[12] M. Wojtyra, J. Fraczek. Solvability of reactions in rigid multibody systems with redundant nonholonomic constraints. Multibody System Dynamics, 2013, 30(2): 153-171.

[13] M. Wojtyra, J. Fraczek. Joint reactions in rigid or flexible body mechanisms with redundant constraints. Bulletin of the Polish Academy of Sciences Technical Sciences, 2012, 60(3): 617-626.

[14] Z.J. Wang, J.T. Yao, Y.D. Xu, et al. Hyperstatic analysis of a fully pre-stressed six-axis force/torque sensor. Mechanism and Machine Theory, 2012, 57: 84-94.

[15] B. Hu, Z. Huang. Kinetostatic model of overconstrained lower mobility parallel manipulators. Nonlinear Dynamics, 2016, 86(1): 309-322.

[16] Z. Huang, Y. Zhao, J.F. Liu. Kinetostatic analysis of 4-R(CRR) parallel manipulator with overconstraints via reciprocal-screw theory. Advances in Mechanical Engineering, 2010, 2010(2): 1652-1660.

[17] J. Zhang, Y.Q Zhao, Y. Jin. Kinetostatic-model-based stiffness analysis of Exechon PKM. Robotics and Computer Integrated Manufacturing: An International Journal of Manufacturing and Product and Process Development, 2016, 37: 208-220.

[18] A. Rezaei, A. Akbarzadeh. Position and stiffness analysis of a new asymmetric 2 PRR-PPR parallel CNC machine. Advanced Robotics, 2013, 27(2): 133-145.

[19] C. Yang, Q.C. Li, Q.H. Chen, et al. Elastostatic stiffness modeling of overconstrained parallel manipulators. 
Mechanism and Machine Theory, 2018, 122: 58-74.

[20] J. Wu, L.P. Wang, L.W. Guan. A study on the effect of structure parameters on the dynamic characteristics of a PRRRP parallel manipulator. Nonlinear Dynamics, 2013, 74: 227-235.

[21] A. Arian, B. Danaei, M. Tale Masouleh. Kinematic and dynamic analyses of tripteron, an over-constrained 3-DOF translational parallel manipulator, through Newton-Euler approach. AUT Journal of Modeling and Simulation, 2018, 50 (1): 61-70.

[22] C. Yang, Q. Chen, J. Tong, et al. Elastostatic stiffness analysis of a 2PUR-PSR overconstrained parallel mechanism. International Journal of Precision Engineering and Manufacturing, 2019, 20: 569-581.

[23] A. Pott. An improved force distribution algorithm for over-constrained cable-driven parallel robots. Mechanisms and Machine Science, 2014, 15: 139-146.

[24] E. Zahariev, J. Cuadrado. Dynamics of mechanisms in overconstrained and singular configurations. Journal of Theoretical and Applied Mechanics, 2011, 41 (1): 3-18.

[25] D.V Sapio, N. Srinivasa. A methodology for controlling motion and constraint forces in holonomically constrained systems. Multibody System Dynamics, 2015, 32 (2): 179-204.

[26] D.S. Zhang, Y.D. Xu, J.T. Yao, et al. Analysis and optimization of a spatial parallel mechanism for a new 5-DOF hybrid serial-parallel manipulator. Chinese Journal of Mechanical Engineering, 2018, 31(3): 54.

[27] A. Müller. Internal preload control of redundantly actuated parallel manipulators-its application to backlash avoiding control. IEEE Transactions on Robotics, 2005, 21(4): 668-677.

[28] J. Wu, J. Wang, L. Wang, et al. Study on the stiffness of a 5-DOF hybrid machine tool with actuation redundancy. Mechanism and Machine Theory, 2009, 44 (2): 289-305.

[29] S.B. Nokleby, R. Fisher, R.P. Podhorodeski, et al. Force capabilities of redundantly-actuated parallel manipulators. Mechanism and Machine Theory, 2005, 40 (5): 578-599.

[30] Y. Zhao, F. Gao. Dynamic performance comparison of the 8PSS redundant parallel manipulator and its non-redundant counterpart - the 6PSS parallel manipulator. Mechanism and Machine Theory, 2009, 44 (5): 991-1008.

[31] J. Wu, J.S. Wang, L.P. Wang, et al. Dynamics and control of a planar 3-DOF parallel manipulator with actuation redundancy. Mechanism and Machine Theory, 2009, 44 (4): 835-849.

[32] X.F. Liu, J.T. Yao, Y.D. Xu, et al. Research of driving force coordination mechanism in parallel manipulator with actuation redundancy and its performance evaluation. Nonlinear Dynamics, 2017, 90 (2): 983-998. 
[33] V. Garg, S.B. Nokleby, J.A. Carretero. Wrench capability analysis of redundantly actuated spatial parallel manipulators. Mechanism and Machine Theory, 2009, 44 (5): 1070-1081.

[34] B. Zi, J. Lin, S. Qian. Localization, obstacle avoidance planning and control of a cooperative cable parallel robot for multiple mobile cranes. Robotics and Computer Integrated Manufacturing, 2015, 34: 105-123.

[35] S. Qian, B. Zi, H. Ding. Dynamics and trajectory tracking control of cooperative multiple mobile cranes. Nonlinear Dynamics, 2016, 83 (1): 89-108.

[36] Y. Lu, Z. Dai, P. Wang. Full forward kinematics of redundant kinematic hybrid manipulator. Applied Mathematical Modelling, 2018, 62 (10): 134-144.

[37] Y. Xu, F.L. Guan. Structure-electronic synthesis design of deployable truss antenna. Aerospace Science and Technology, 2013, 26 (1): 259-267.

[38] T.J. Li. Deployment analysis and control of deployable space antenna. Aerospace Science and Technology, 2012, 18: 42-47.

[39] M. Jin, A. Kazuhide, S. Yumi, et al. Deployment analysis of large space antenna using flexible multibody dynamics simulation. Acta Astronautica, 2000, 47 (1): 19-26.

[40] R.Q. Liu, D.K. Tian, Z.Q. Deng, et al. Kinematics modeling and driving spring design of truss stucture for deployable truss antenna. 2011 International Conference on Advanced Materials and Engineering Materials, Shenyang, Liaoning, China, November 22-24, 2011: 1337-1341.

[41] Y.D. Xu, Z. Jiang, Z.J. Ju, et al. Force analysis of the redundantly actuated parallel mechanism 2 RPR $+P$ considering different control methodologies. Robotics and Autonomous Systems, 2021, (1): 103783. 